Bull. Korean Math. Soc. 52 (2015), No. 6, pp. 1963-1971

http://dx.doi.org/10.4134/BKMS.2015.52.6.1963

\title{
MAPPING PRESERVING NUMERICAL RANGE OF OPERATOR PRODUCTS ON $C^{*}$-ALGEBRAS
}

\author{
Mohamed Mabrouk
}

\begin{abstract}
Let $\mathcal{A}$ and $\mathcal{B}$ be two unital $C^{*}$-algebras. Denote by $W(a)$ the numerical range of an element $a \in \mathcal{A}$. We show that the condition $W(a x)=W(b x), \forall x \in \mathcal{A}$ implies that $a=b$. Using this, among other results, it is proved that if $\phi: \mathcal{A} \rightarrow \mathcal{B}$ is a surjective map such that $W(\phi(a) \phi(b) \phi(c))=W(a b c)$ for all $a, b$ and $c \in \mathcal{A}$, then $\phi(1) \in Z(B)$ and the map $\psi=\phi(1)^{2} \phi$ is multiplicative.
\end{abstract}

\section{Introduction}

Let $\mathcal{A}$ be a $C^{*}$-algebra with unit 1 and let $S(\mathcal{A})$ be the state space of $\mathcal{A}$, i.e., $S(\mathcal{A})=\left\{\varphi \in \mathcal{A}^{\prime}: \varphi \geq 0, \varphi(1)=1\right\}$ (here $\mathcal{A}^{\prime}$ is the topological dual of $\mathcal{A}$ ). For each $a \in \mathcal{A}$, the algebraic numerical range $V(a)$ and numerical radius $v(a)$ are defined by

$$
V(a)=\{f(a): f \in S(\mathcal{A})\} \text { and } v(a)=\sup _{z \in V(a)}|z| .
$$

By the Gelefand-Naimark theorem, every $C^{*}$-algebra may be viewed as a closed *-subalgebra of $B(H)$ where $B(H)$ denotes the algebra of all bounded linear operators acting on a Hilbert space $H$. It is well known that $V(a)$ is the closure of $W(a)$ and $v(a)=w(a)=\sup _{\lambda \in W(a)}|\lambda|$, where $W(a)=\{(a t, t): t \in H,\|t\|=1\}$ and $($,$) denotes the inner product. Here W(a)$ is called the usual numerical range of the operator $a$.

In the last few decades, there has been a considerable interest in the problem of characterization of maps that preserves the numerical range or the numerical radius, see for instance the papers $[4,12,13,15]$ and the references therein. Notice that, based on the aforesaid, preserving the usual numerical range $W$ implies the preservation of the spacial numerical range $V$. Therefore, we will concentrate our study henceforth on $W$. Recently, Hou and Di described in [9] surjective maps on the algebra $B(H)$ which preserves the numerical range of

Received September 24, 2014; Revised April 28, 2015.

2010 Mathematics Subject Classification. 15A86, 46L05, 47A12, 47B49.

Key words and phrases. $C^{*}$-algebras, numerical range, preserving the numerical range. 
the product. Namely, they characterized surjective mappings which satisfy one of the following conditions

$$
\begin{aligned}
W(\phi(a) \phi(b)) & =W(a b), \\
W\left(\phi(a)^{*} \phi(b)\right) & =W\left(a^{*} b\right), \\
W(\phi(a) \phi(b) \phi(a)) & =W(a b a),
\end{aligned}
$$

for every $a$ and $b$ in $B(H)$. In this paper, we extend these results by completely describing additive and surjective maps $\phi: \mathcal{A} \rightarrow \mathcal{B}$ between $C^{*}$-algebras satisfying (1a) or (1b) for every $a, b \in \mathcal{A}$. Concerning the condition (1c), we consider a more general case. More precisely, we show that if $\phi$ is surjective and satisfy $W(\phi(a) \phi(b) \phi(c))=W(a b c), \forall a, b$ and $c \in \mathcal{A}$ (without the additivity assumption), then the map $\psi=\phi(1)^{2} \phi$ is multiplicative and preserves the set of self-adjoint elements. It is worth noticing that our proofs differ from those of [7] and [9] since we do not assume that $\mathcal{A}$ contains rank one operators. At last, observe that the proof we put forward is much simpler.

The outline of the paper is as follows. Firstly, we show that if $a$ and $b$ in $\mathcal{A}$ are such that $W(a x)=W(b x)$ for every $x \in \mathcal{A}$, then the two operators $a$ and $b$ coincide. This result is used several times in our proofs. Namely, it helps us to show that if $\phi$ is additive and satisfies $(1 \mathrm{a})$ or $(1 \mathrm{~b})$, then $\phi(1) \in \mathcal{Z}(\mathcal{B})$, where $Z(\mathcal{B})$ stands for the center of $\mathcal{B}$, and $\phi(1) \phi$ is a Jordan $*$-isomorphism. This characterization also allows us to show that if a map $\phi$ is surjective and satisfies $W(a b c)=W(\phi(a) \phi(b) \phi(c))$ whenever $a, b$ and $c$ are in $\mathcal{A}$, then the map $\phi(1)^{2} \phi$ is multiplicative and therefore $\phi$ has standard forms when $\mathcal{A}$ and $\mathcal{B}$ are the algebras of all bounded linear operators acting on a Hilbert space.

\section{Preliminaries}

In this section, we collect some properties of the numerical range needed in the sequel. Let two unital $C^{*}$-algebras $\mathcal{A} \subset B(H)$ and $\mathcal{B} \subset B(K)$ be given. By $\operatorname{Sp}(a)$ (resp. $r(a)$ ) we denote the spectrum (resp. the spectral radius) of an element $a \in \mathcal{A}$. Since it does not lead to misunderstanding, we shall denote the norms in both algebras by the same symbols $\|\cdot\|$. We denote by $\mathcal{H}(\mathcal{A})$ the set of self adjoint elements defined by $\mathcal{H}(\mathcal{A})=\left\{a \in \mathcal{A}: a=a^{*}\right\}$. It is well-known that $a \in \mathcal{H}(\mathcal{A})$ if and only if $W(a) \subset \mathbb{R}$. Further, an element $a \in \mathcal{A}$ is positive if and only if $W(a) \subset \mathbb{R}_{+}$(or equivalently $a=a^{*}$ and $\operatorname{Sp}(a) \subset \mathbb{R}_{+}$), where $\mathbb{R}_{+}$denotes the set of positive real numbers. In the case where $\mathcal{A}=C(K)$ for some Hausdorff compact space $K$ we have $W(a) \subset V(a)=\operatorname{co}(a(K))$ for each $a \in C(K)$, see [16, Theorem 6]. Here co stands for closed convex hull. We summarize some other basic properties of the numerical range on the following lemma. One may see $[2,8]$ for more information.

\section{Lemma 2.1 .}

(i) $\|a\|=w(a)=r(a)$ for every $a \in \mathcal{A}$ such that $a a^{*}=a^{*} a$.

(ii) $W(a)=\{\lambda\} \Longleftrightarrow a=\lambda 1$, for every $a \in \mathcal{A}$ and $\lambda \in \mathbb{C}$. 
Finally, recall that a linear map $\psi: \mathcal{A} \rightarrow \mathcal{B}$ is called unital if $\psi(1)=1$, and it is said to be a Jordan homomorphism if $\psi\left(a^{2}\right)=\psi(a)^{2}$ for all $a \in \mathcal{A}$. Equivalently, the map $\psi$ is a Jordan homomorphism if and only if $\psi(a b+b a)=$ $\psi(a) \psi(b)+\psi(b) \psi(a)$ for all $a$ and $b$ in $\mathcal{A}$. We also recall that the map $\psi$ is said to be self-adjoint provided that $\psi\left(a^{*}\right)=\psi(a)^{*}$ for all $a \in \mathcal{A}$. Self-adjoint Jordan homomorphisms are called Jordan *-homomorphisms, and by a Jordan $*$-isomorphism, we mean a bijective $*$-homomorphism.

\section{Main results and proofs}

We start with the following introductory results, which may be of independent interest. We give a characterization of elements $a, b \in \mathcal{A}$ satisfying $W(a x)=W(b x), \forall x \in \mathcal{A}$ or $w(a x)=w(b x), \forall x \in \mathcal{H}(\mathcal{A})$. It is worth observing that the authors in [3] have recently considered the question whether the equality $\operatorname{Sp}(a x)=\operatorname{Sp}(b x)$ for every $x \in \mathcal{A}$, where $a, b \in \mathcal{A}$ are fixed elements, implies $a=b$. An affirmative answer has been obtained for some classes of algebras, including $C^{*}$-algebras.

We begin with the following proposition which gives necessary conditions which ensure that $a=b$ if $w(a x)=w(b x), \forall x \in \mathcal{H}(\mathcal{A})$. The argument of the proof is borrowed from [11, Lemma 3.4] by slight some modifications. We present it here for the sake of completeness.

Proposition 3.1. Let $\mathcal{A}$ be an unital $C^{*}$-algebra and $a, b \in \mathcal{A}$ be two positive elements such that $a b=b a$. If $w(a x)=w(b x)$ for every $x \in \mathcal{H}(\mathcal{A})$, then $a=b$.

Proof. Let $\mathcal{B}$ be the unital $C^{*}$-algebra $\mathcal{B}$ generated by $a$ and $b$. Since, $a b=b a$, this algebra is commutative. Henceforth, without loss of generality we may suppose that $\mathcal{A}$ is a commutative $C^{*}$-algebra. On the other hand, it is well known that every positive element in a $C^{*}$-algebra has unique square root, then to prove that $a=b$, it suffices to show that $a^{2}=b^{2}$. Suppose to the contrary that $a^{2} \neq b^{2}$. Since $a^{2}-b^{2}$ is self-adjoint, there exists a non-zero $\beta \in \operatorname{Sp}\left(a^{2}-b^{2}\right)$. We may assume that $\beta>0$ (otherwise, we could replace $a^{2}-b^{2}$ by $\left.b^{2}-a^{2}\right)$. Let $\alpha=\frac{1}{2} \sup \operatorname{Sp}\left(a^{2}-b^{2}\right)>0$, and consider the real valued continuous function $f$ defined on the spectrum of $a^{2}-b^{2}$ such that $f(2 \alpha)=1,0 \leq f(\lambda) \leq 1, \forall \lambda \in \operatorname{Sp}\left(a^{2}-b^{2}\right)$ and $f(\lambda)=0 \Longleftrightarrow \lambda \leq \alpha$. Put $x_{1}=f\left(a^{2}-b^{2}\right)$ and $g(\lambda)=\lambda f(\lambda)^{2}, \forall \lambda \in \operatorname{Sp}\left(a^{2}-b^{2}\right)$. So, using functional calculus (see [6, Theorem 2.9]) and the fact that $x_{1}\left(a^{2}-b^{2}\right) x_{1}$ is self adjoint, we get $w\left(x_{1}\left(a^{2}-b^{2}\right) x_{1}\right)=r\left(x_{1}\left(a^{2}-b^{2}\right) x_{1}\right)=\sup _{\lambda \in \operatorname{Sp}\left(a^{2}-b^{2}\right)}|g(\lambda)|=2 \alpha$. In addition by using the same argument, it is easily shown that $x_{1}\left(a^{2}-b^{2}\right) x_{1} \geq$ $\alpha x_{1}^{2}$. Now for $t \in H$ such that $\|t\|=1$, let us define the positive linear form $\varphi_{t}$ by $\varphi_{t}(a)=(a t, t), \forall a \in \mathcal{A}$. Since $\|b\| x_{1}^{2}-x_{1} b^{2} x_{1}$ is positive, we have $\|b\| \varphi_{t}\left(x_{1}^{2}\right) \geq \varphi_{t}\left(x_{1} b^{2} x_{1}\right) \geq 0$. On account of $x_{1}\left(a^{2}-b^{2}\right) x_{1} \geq \alpha x_{1}^{2}$, it follows that $\varphi_{t}\left(x_{1} a^{2} x_{1}\right) \geq\left(1+\frac{\alpha}{\|b\|}\right) \varphi_{t}\left(x_{1} b^{2} x_{1}\right)$. Since $0 \leq \varphi_{t}\left(x_{1} a^{2} x_{1}\right) \leq w\left(x_{1} a^{2} x_{1}\right)$, we infer that $w\left(x_{1} a^{2} x_{1}\right) \geq\left(1+\frac{\alpha}{\|b\|}\right) w\left(x_{1} b^{2} x_{1}\right)$. Accordingly $\left\|a x_{1}\right\| \geq \sqrt{1+\frac{\alpha}{\|b\|}}\left\|b x_{1}\right\|>$ $\left\|b x_{1}\right\|$. This obviously contradicts the hypothesis of the proposition, since $x_{1} \in$ 
$\mathcal{H}(\mathcal{A})$ and by Lemma 2.1 , we have $\left\|a x_{1}\right\|=w\left(a x_{1}\right)=w\left(b x_{1}\right)=\left\|b x_{1}\right\|$. Thus $a=b$ as desired.

The next two propositions are crucial for the rest of the paper. They give a characterization of elements $a, b \in \mathcal{A}$ satisfying $W(a x)=W(b x)$ for every $x \in \mathcal{A}($ or $\mathcal{H}(\mathcal{A}))$.

Proposition 3.2. Let $\mathcal{A}$ be an unital $C^{*}$-algebra and $a, b \in \mathcal{A}$. If $W(a x)=$ $W(b x), \forall x \in \mathcal{A}$, then $a=b$.

Proof. Firstly, assume that $a=a^{*}$. Since $W(b)=W(a) \subset \mathbb{R}$, then $b^{*}=b$. On the other hand, by observing that $W\left(a^{2}\right)=W(b a)$ and the fact that $a^{2}$ is self adjoint, we infer that $(b a)^{*}=b a$. By taking into account that $(b a)^{*}=a^{*} b^{*}$ and that $a$ and $b$ are self adjoint, we get $a b=b a$. We prove now that $a=b$. Let $\mathcal{B}$ be the $C^{*}$-algebra $\mathcal{B}$ generated by $a$ and $b$. Since $a$ and $b$ are self adjoint and satisfy $a b=b a$, this algebra is commutative. Hence, it can be identified with $C(K)$, the algebra of all continuous functions on a compact Hausdorff space $K$. Observe also that $W(a x)=W(b x), \forall x \in \mathcal{B}$. We claim that the two functions $a$ and $b$ have the same sign (both positive, or both negative on $K$ ). Indeed, assuming that there exists $t_{1} \in K$ such that $a\left(t_{1}\right)>0$ and $b\left(t_{1}\right)<0$. Therefore there exists an open set $U_{1}$ such that $a(t)>0$ and $b(t)<0$ for all $t \in U_{1}$. By Urysohn's lemma, one can find a continuous function $c_{1}: K \rightarrow[0,1]$ satisfying $c\left(t_{1}\right)=1$ and $\operatorname{supp}(c) \subset U_{1}$. On the other hand $W(a c) \subset \operatorname{co}(a c(K))$ and $W(b c) \subset \operatorname{co}(b c(K))$. Then, we get $W(a c) \subset[0,+\infty)$ and $W(b c) \subset(-\infty, 0]$. Observe that the case where $W(a c)=W(b c)=\{0\}$, does not occur since $a c \neq 0$. Therefore $W(a c) \neq W(b c)$, contrary to our assumption. Thus $a$ and $b$ have the same sign as suggested above. Consequently, without loss of generality, we can suppose that $a, b$ are positive on $U$. From the condition $W(a x)=W(b x), \forall x \in \mathcal{B}$, we infer that $w(a x)=w(b x), \forall x \in \mathcal{B}$. It follows from Proposition 3.1 that $a=b$. We return now to the general case; i.e., if $a \in \mathcal{A}$ is arbitrary. Observe that, by assumption, we have, $W\left(a a^{*} x\right)=W\left(b a^{*} x\right)$ and $W\left(a b^{*} x\right)=W\left(b b^{*} x\right), \forall x \in \mathcal{A}$. Based on the aforesaid, we infer that $a a^{*}=b a^{*}$ and $a b^{*}=b b^{*}$. Accordingly, $(a-b)\left(a^{*}-b^{*}\right)=0$, which implies that $a=b$. This ends the proof.

Proposition 3.3. If $\mathcal{A}$ is a unital $C^{*}$-algebra and a and $b \in \mathcal{H}(\mathcal{A})$. If $W(a x)=$ $W(x b), \forall x \in \mathcal{H}(\mathcal{A})$ or $W(a x)=W(b x), \forall x \in \mathcal{H}(\mathcal{A})$, then $a=b$.

Proof. We give the proof for the condition $W(a x)=W(x b), \forall x \in \mathcal{H}(\mathcal{A})$. For the other condition the proof is similar. By using a similar reasoning as above, we can easily prove that $a b=b a$. Considering the commutative $C^{*}$-algebras $\mathcal{B}$ generated by $a, b$ and by taking into account that $W(a x)=W(b x), \forall x \in \mathcal{H}(\mathcal{B})$. By a similar reasoning as in the proof of the above proposition, we can show that $a$ and $b$ are either both positive or negative. We infer by means of Proposition 3.1 that $a=b$. 
Remark 3.4. The results of Propositions 3.2 and 3.3 are still valid if we replace the numerical range by its closure. That is if two elements $a$ and $b$ satisfy $V(a x)=V(b x)$ for every $x \in \mathcal{A}($ or in $\mathcal{H}(\mathcal{A}))$, then a similar argument can be used to show that $a=b$.

At this juncture, we are in a position to characterize surjective mapping satisfying

$$
\begin{aligned}
& W(\phi(a) \phi(b) \phi(c))=W(a b c) \text { for all } a, b \text { and } c \in \mathcal{A}, \\
& W(\phi(a) \phi(b) \phi(c))=W(a b c) \text { for all } a, b \text { and } c \in \mathcal{H}(\mathcal{A}) .
\end{aligned}
$$

Theorem 3.5. Let $\mathcal{A}$ and $\mathcal{B}$ be two unital $C^{*}$-algebras. Let $\phi: \mathcal{A} \rightarrow \mathcal{B}$ be a surjective mapping satisfying (2a). Then $\phi(1) \in Z(\mathcal{B}), \phi(1)^{3}=1$, and $\phi$ satisfies $\phi(a b)=\phi(1)^{2} \phi(a) \phi(b)$ for all $a$ and $b \in \mathcal{A}$. In particular, the mapping $\psi=\phi(1)^{2} \phi$ is multiplicative and preserves self-adjoint elements (i.e., $\psi(a) \in \mathcal{H}(\mathcal{B})$ whenever $a \in \mathcal{H}(\mathcal{A}))$.

Proof. Set $u=\phi(1)$. Take $a=b=c=1$ in (2a), we obtain $W\left(u^{3}\right)=W(1)=$ $\{1\}$. Thus $u^{3}=1$ and hence $u$ is invertible. Given $a, b \in \mathcal{A}$ such that $\phi(a)=$ $\phi(b)$. By $(2 \mathrm{a})$, we have $W(a c)=W(u \phi(a) \phi(c))=W(u \phi(b) \phi(c))=W(b c)$ for every $c \in \mathcal{A}$. By Proposition 3.2, we infer that $a=b$ and $\phi$ is bijective as desired. Also, we have $W(u \phi(a) \phi(b))=W(1 a b)=W(a 1 b)=W(\phi(a) u \phi(b))$. Thus we get $W(u \phi(a) \phi(b))=W(\phi(a) u \phi(b)), \forall b \in \mathcal{A}$. Since $\phi$ is a bijection and on account of Proposition 3.2, we get $u \phi(a)=\phi(a) u, \forall a \in \mathcal{A}$. That is to say that $u \in Z(\mathcal{B})$. To end the proof, observe that

$$
\begin{aligned}
W(\phi(a) \phi(b) \phi(c)) & =W(a b c)=W(1(a b) c) \\
& =W(u \phi(a b) \phi(c)), \forall c \in \mathcal{A} .
\end{aligned}
$$

By recalling that $\phi$ is bijective, again Proposition 3.2 implies that $\phi(a b)=$ $u^{-1} \phi(a) \phi(b)=u^{2} \phi(a) \phi(b)$. Now, put $\psi=u^{2} \phi$. We have

$$
\psi(a) \psi(b)=u^{2} \phi(a) u^{2} \phi(b)=u \phi(a) \phi(b)=u^{2} \phi(a b)=\psi(a b) .
$$

Finally, observe that $\phi$ preserves self-adjoint elements because for every self adjoint element $a \in \mathcal{A}$, we have $W(\psi(a))=W\left(\phi(1)^{2} \phi(a)\right)=W(a) \subset \mathbb{R}$. The proof is thus complete.

Corollary 3.6. Let $\mathcal{A}$ and $\mathcal{B}$ be two unital $C^{*}$-algebras. Let $\phi: \mathcal{A} \rightarrow \mathcal{B}$ be a surjective mapping satisfying

$$
W\left(\phi\left(a_{1}\right) \phi\left(a_{2}\right) \cdots \phi\left(a_{p}\right)\right)=W\left(a_{1} a_{2} \cdots a_{p}\right) \text { for all } a_{1} a_{2} \cdots a_{p} \in \mathcal{A}
$$

for some integer $p \in \mathbb{N}$ with $p \geq 3$. Then $\phi(1) \in Z(\mathcal{B}), \phi(1)^{p}=1$ and $\phi(1)^{p-1} \phi\left(a_{1} a_{2} \cdots a_{p}\right)=\phi\left(a_{1}\right) \phi\left(a_{2}\right) \cdots \phi\left(a_{p}\right)$ for all $a_{1} a_{2} \cdots a_{p} \in \mathcal{A}$.

Proof. It is obvious that $\phi(1)^{p}=1$. If $\phi(a)=\phi(b)$ for some $a, b \in \mathcal{A}$. Since $W\left(\phi(a) \phi(x) \phi(1)^{p-2}\right)=W\left(\phi(b) \phi(x) \phi(1)^{p-2}\right)$, by (3), it yields that $W(a x)=$ 
$W(b x)$ for every $x \in \mathcal{A}$. By Proposition 3.2, we get $a=b$. Hence $\phi$ is a bijection. Define $\psi=\phi(1)^{p-1} \phi$. We see that $\psi(1)=1$ and

$$
\psi(a) \psi(b) \psi(c)=\phi(1)^{p-1} \phi(a) \phi(1)^{p-1} \phi(b) \phi(1)^{p-1} \phi(c)=\phi(1)^{p-3} \phi(a) \phi(b) \phi(c) .
$$

From (3), we can deduce that $W(\psi(a) \psi(b) \psi(c))=W(a b c), \forall a, b, c \in \mathcal{A}$. By Theorem 3.5, the results follows.

Based, on Theorem 3.5, we know that if $\phi$ satisfy (2a), then the mapping $\psi=\phi(1)^{2} \phi$ is multiplicative. The question of when a multiplicative map is additive was attacked by several authors. For instance, if $\psi$ is a bijective map on a standard operator algebra, Molnàr showed in [14] that if $\phi$ satisfies $\psi(A B A)=\psi(A) \psi(B) \psi(A)$, then $\psi$ is additive. Hence, based on the aforesaid, when the algebras $\mathcal{A}$ and $\mathcal{B}$ are the algebras of all bounded linear operators acting on some Hilbert spaces, Theorem 3.5 can be refined as follows.

Corollary 3.7. Let $H$ and $K$ be complex Hilbert spaces and let $\phi: B(H) \rightarrow$ $B(K)$ be a surjective map (without the assumption of additivity). Then $\phi$ satisfies Eq. (2a) if and only if there is a unitary operator $U: H \rightarrow K$ such that $\phi$ is of the form $\phi(A)=\varepsilon U A U^{*}$ for all $A \in B(H)$, where $\varepsilon^{3}=1$.

Proof. Checking the 'if' part is straightforward, and we therefore will only deal with the 'only if' part. Assume that $\phi$ satisfies (2a). By Theorem 3.5, we have that $\phi(1) \in Z(B(K))$ and $\phi(1)^{3}=1$. Since the algebra $B(K)$ has a trivial center, then $u=\phi(1)=\varepsilon \cdot 1$, where $\varepsilon$ is a complex number such that $\varepsilon^{3}=1$. Also according to Theorem 3.5, the map $\psi=u^{2} \phi$, is multiplicative and $\psi(1)=1$. Consequently, by [14] it is additive. Finally, we have shown that $\psi$ is an algebra isomorphism which preserves self-adjoint elements. Thus, by [4] $\psi$ takes the following form: $\psi(A)=U A U^{*}$ for all $A \in B(H)$ where $U$ is unitary.

For mapping $\phi: \mathcal{H}(\mathcal{A}) \rightarrow \mathcal{H}(\mathcal{B})$ satisfying $(2 \mathrm{~b})$, we have a similar result which follows.

Theorem 3.8. Let $\mathcal{A}$ and $\mathcal{B}$ be two unital $C^{*}$-algebras. Let $\phi: \mathcal{H}(\mathcal{A}) \rightarrow \mathcal{H}(\mathcal{B})$ be a surjective mapping satisfying $(2 \mathrm{~b})$. Then $\phi(1) \in Z(\mathcal{H}(\mathcal{B})), \phi(1)^{3}=1$, and $\phi=\phi(1) \psi$, where $\psi$ is multiplicative.

Proof. The proof is similar to that of Theorem 3.5 by invoking Proposition 3.3. The details are omitted.

As a special case of Theorem 3.8 we derive the following result.

Theorem 3.9. Consider the case where $\mathcal{A}=B(H)$ and $\mathcal{B}=B(K)$ for some complex Hilbert spaces $H$ and $K$. Let $\phi: \mathcal{H}(\mathcal{A}) \rightarrow \mathcal{H}(\mathcal{B})$ be a surjective map. Then, $\phi$ satisfies $(2 \mathrm{~b})$ if and only if there exists a unitary or conjugate unitary operator $U$ and $\varepsilon \in \mathbb{C}$ such that $\phi(A)=\varepsilon U A U^{*}$ for all $A \in \mathcal{H}(\mathcal{A})$ and $\varepsilon^{3}=1$. 
Proof. The sufficiency is easy to see. Indeed, this follows from the well-known fact that if $U$ is a unitary or conjugate unitary operator, then $W\left(U A U^{*}\right)=$ $W(A)$ for every $A \in \mathcal{A}$. Conversely, suppose that $\phi$ satisfies Eq. (2b) for every $A \in \mathcal{H}(\mathcal{A})$. Theorem 3.8, implies that $\psi=\phi(1)^{2} \phi$ is multiplicative and $\psi(1)=1$. Therefore, by $[1$, Theorem 2.1] there exists a unitary or conjugate unitary operator $U$ such that $\psi(A)=U A U^{*}$ for all $A \in \mathcal{H}(\mathcal{A})$. To end the proof observe that $\phi=u \psi=\phi(1) \psi$ and in particular $\phi$ is linear. Since, by Theorem 3.8 we have $\phi(1) \in Z(\mathcal{H}(\mathcal{B}))$, we infer that $\phi(1) \in Z(\mathcal{B})$. Therefore, $\phi(1)=\varepsilon .1$, where $\varepsilon$ is a complex number such that $\varepsilon^{3}=1$. Thus, completing the proof.

We give now the following theorem which characterizes surjective maps satisfying (1a) in the case of $C^{*}$-algebras. This result has been also proved in [7, Theorem 2.1.] for the Hilbert space operators case but without the extra condition that $\phi$ is additive. It would be interesting to remove the additive assumption in Theorem 3.10 below. We are not able to do that at present.

Theorem 3.10. Let $\mathcal{A}$ and $\mathcal{B}$ be two unital $C^{*}$-algebra and $\phi: \mathcal{A} \rightarrow \mathcal{B}$ be a surjective and additive map satisfying (1a). Then $\phi$ is a Jordan $*$-isomorphism followed by a left multiplication by a fixed element $u \in Z(\mathcal{B})$ with $u^{2}=1$, where $Z(\mathcal{B})$ stands for the center of $\mathcal{B}$.

Proof. Firstly, we prove that $\phi$ is bijective. It suffice to show that it is injective. Let $a, b \in \mathcal{A}$ such that $\phi(a)=\phi(b)$. By (1a), we have $W(\phi(a) \phi(c))=W(a c)=$ $W(\phi(b) \phi(c))=W(b c), \forall c \in \mathcal{A}$. By Proposition 3.2, it yields that $a=b$. Hence we have proved that $\phi$ is injective. Take $a=b=1$ in (1a), we obtain $W\left(\phi(1)^{2}\right)=W(1)=\{1\}$. Whence $\phi(1)$ is invertible and $\phi(1)^{2}=1$. We show now that $\phi$ is linear. Let $\lambda \in \mathbb{C}$. By (1a) we have

$$
\begin{aligned}
W(\lambda \phi(a) \phi(b)) & =\lambda W(\phi(a) \phi(b))=\lambda W(a b) \\
& =W(\lambda a) b)=W(\phi(\lambda a) \phi(b)), \forall a, b \in \mathcal{A} .
\end{aligned}
$$

Whence, by Proposition 3.2, it yields that $\phi(\lambda a)=\lambda \phi(a), \forall a \in \mathcal{A}$. Since $\phi$ is additive, we infer that $\phi$ is a linear bijection. Now, take $a, b \in \mathcal{A}$ such that $a b=0$. By (1a), yields that $\phi(a) \phi(b)=0$. Hence [5, Lemma 4.4], implies that $\phi(1) \phi(a)=\phi(a) \phi(1), \forall a \in \mathcal{A}$. Together with the bijectivity of $\phi$, this implies that $\phi(1) \in Z(\mathcal{B})$.

Finally, we show that $\phi$ has the asserted form. Set $\psi=u \phi$. It suffices to show that $\psi$ is $C^{*}$-isomorphism. It is obvious that $\psi(1)=u^{2}=1$ and $W(\psi(a) \psi(b))=W(a b), \forall a, b \in \mathcal{A}$. Thus, we have proved that $\psi$ is a linear isomorphism satisfying $W(\psi(a))=W(a)$ for every $a \in \mathcal{A}$. By [15, Theorem 3.1 , the result follows.

Finally, we turn to the second type of preserver problems involving involution. We have the following result. 
Theorem 3.11. Let $\mathcal{A}$ and $\mathcal{B}$ be two unital $C^{*}$-algebra and $\phi: \mathcal{A} \rightarrow \mathcal{B}$ be a surjective and additive map satisfying (1b). Then, $\phi(1)$ is unitary and $\phi=$ $\phi(1) \psi$, where $\psi$ is a Jordan *-isomorphism.

Proof. Firstly, observe that by (1b), we have

$$
\|\phi(a)\|^{2}=\left\|\phi(a) \phi(a)^{*}\right\|=w\left(\phi(a) \phi(a)^{*}\right)=w\left(a a^{*}\right)=\left\|a a^{*}\right\|^{2}, \forall a \in \mathcal{A} .
$$

Taking the square root, we obtain $\|\phi(a)\|=\|a\|$, which yields that $\phi$ is an isometry and hence a bijection. Now, let $\lambda \in \mathbb{C}$ and $a \in \mathcal{A}$. For all $b \in \mathcal{A}$, we have

$$
\begin{aligned}
W\left((\lambda \phi(a))^{*} \phi(b)\right) & =\bar{\lambda} W\left(\phi(a)^{*} \phi(b)\right)=\bar{\lambda} W\left(a^{*} b\right) \\
& =W\left((\lambda a)^{*} b\right)=W\left((\phi(\lambda a))^{*} \phi(b)\right) .
\end{aligned}
$$

Using Proposition 3.2, we infer that $(\lambda \phi(a))^{*}=(\phi(\lambda a))^{*}$. Accordingly, $\lambda \phi(a)=$ $\phi(\lambda a)$. In consequence of this, $\phi$ is a linear bijection. Thus, we have proved that $\phi$ is a linear isomorphism between two $C^{*}$-algebras which are isometric. By [10, Theorem 7], $\phi$ is a Jordan $*$-isomorphism followed by left multiplication by the fixed unitary operator $\phi(1)$.

Acknowledgements. The author wishes to express his thanks to the referee for carefully reading the paper and suggestions.

\section{References}

[1] R. An and J. Hou, Additivity of Jordan multiplicative maps on Jordan operator algebras, Taiwanese J. Math. 10 (2006), no. 1, 45-64.

[2] F. F. Bonsall and J. Duncan, Numerical ranges of operators on normed spaces and elements of normed algebras, Cambridge Univ. Press, London, 1971.

[3] M. Bresar and S. Spela, Determining elements in Banach algebras through spectral properties, J. Math. Anal. Appl. 393 (2012), no. 1, 144-150.

[4] J. T. Chan, Numerical radius preserving operators on $B(H)$, Proc. Amer. Math. Soc. 123 (1995), no. 5, 1437-1439.

[5] M. A. Chebotar, W. F. Ke, P. K. Lee, and N. C. Wong, Mappings preserving zero products, Studia Math. 155 (2003), no. 1, 77-94.

[6] J. B. Conway, A Course in Functional Analysis, Springer, 1990.

[7] H. L. Gau and C. K. Li, $C^{*}$-isomorphisms, Jordan isomorphisms, and numerical range preserving maps, Proc. Amer. Math. Soc. 135 (2007), no. 9, 2907-2914.

[8] P. R. Halmos, A Hilbert Space Problem Book, 2nd ed., Springer, New York, 1982.

[9] J. Hou and Q. Di, Maps preserving numerical ranges of operator products, Proc. Amer. Math. Soc. 134 (2006), no. 5, 1435-1446.

[10] R. V. Kadisson, Isometries of operator algebras, Ann. of Math. 54 (1951), no. 2, 325338.

[11] E. C. Lance, Unitary operators on Hilbert $C^{*}$-modules, Bull. London Math. Soc. 4 (1994), no. 4, 363-366.

[12] C. K. Li, A survey on linear preservers of numerical ranges and radii, Taiwanese J. Math. 5 (2001), no. 3, 477-496.

[13] C. K. Li and E. Poon, Maps preserving the joint numerical radius distance of operators, Linear Algebra Appl. 437 (2012), no. 5, 1194-1204.

[14] L. Molnár, On isomorphisms of standard operator algebras, Studia Math. 142 (2000), no. 3, 295-302. 
MAPPING PRESERVING NUMERICAL RANGE OF OPERATOR PRODUCTS 1971

[15] V. Pellegrini, Numerical range preserving operators on a Banach algebra, Studia Math. 54 (1975), no. 2, 143-147.

[16] J. G. Stampfli and J. P. Williams, Growth conditions and the numerical range in a Banach algebras, Tohoku Math. J. 20 (1968), 417-424.

Department of Mathematics

College of Applied Sciences

P. O. Box 715, МаKкан 21955, KSA

AND

Department of Mathematics

FACUlty of SCIENCES

GABÈS - RIADH CITY - ZIRIg 6072 - TUNISIA

E-mail address: msmabrouk@uqu.edu.sa 